\title{
LA META: SEGUIR CRECIENDO
}

Comienza el ciclo 2016 de la Revista de la Sociedad Argentina de Diabetes (SAD) y nos encuentra avanzando un peldaño sobre las metas comentadas en el primer artículo editorial de 2015.

La buena noticia inicial es que en este último año hemos progresado en la indexación, dado que la Revista de la SAD en papel ha sido aceptada e incluida en el catálogo de la base de datos Latindex (Ver: http://www.latindex.org/latindex/ficha?folio=11448).

La evaluación realizada por los responsables del Centro Argentino de Información Científica y Tecnológica (CAICYT) dependiente del Consejo Nacional de Investigaciones Científicas y Técnicas (CONICET) nos calificó con un cumplimiento casi perfecto, de 32 sobre 33 puntos, por la importancia de sumar artículos originales de investigación en forma regular. Si bien la figuración de la Revista es sólo en catálogo, constituye un segundo paso importante luego de haber sido incorporada durante 2014 en la Base de Datos Lilacs (Literatura Latinoamericana y del Caribe en Ciencias de la Salud) coordinada por BIREME (OPS/OMS) lo que facilita la presentación de la Revista en otras bases de datos científicas.

Con respecto a la continuidad del proceso de indexación, estamos en camino de avanzar con SciELO (Scientific Electronic Library Online) y Núcleo Básico de Revistas Científicas Argentinas y, a más largo plazo, el objetivo es lograr la inclusión de la Revista en MEDLINE, que como sabemos constituye la principal base de datos de citas bibliográficas biomédicas dependiente de la Biblioteca Nacional de Medicina de los Estados Unidos (NLM). Esta noticia no sólo jerarquiza a la SAD y a su Revista sino que también le brinda a los investigadores básicos y clínicos del área de la diabetes la posibilidad de difundir sus publicaciones con un mayor impacto a un universo creciente de lectores.

En 2015 se publicaron tres ediciones científicas de 3.000 ejemplares cada una, y dos especiales correspondientes a las Jornadas SAD-FASEN (1.500 ejemplares) y a las Jornadas de Graduados (2.000). Recordamos que se encuentran disponibles en la página web de la SAD, con acceso exclu- sivo a los socios, todas las publicaciones en formato completo desde el año 2011.

Durante 2016 iniciaremos dos nuevos segmentos en la revista que, por su temática y contenido, ayudarán a difundir entre los socios y la comunidad médica información relevante de la investigación local en diabetes. Por una parte, se publicará la línea de trabajo de distintos grupos de investigación y sus proyectos a fin de que los conozcamos y que potenciales colegas interesados puedan profundizar o ampliar sus preferencias y, por otra parte, los perfiles de quienes han actuado y actúan como verdaderos maestros de la Diabetología y sirven con su acción y presencia como estímulo de varias generaciones de médicos en el país y la región. En este número invitamos al Prof. Dr. Juan José Gagliardino a que inicie la sección y nos relate su actividad desde sus comienzos. No dudamos que les resultará tan inspirador como a nosotros.

Desde la Dirección de la Revista queremos compartir un gran motivo de orgullo y honrar a quien fue Director de esta publicación así como también Director de la Escuela de Graduados y Presidente de nuestra Sociedad. El Académico Prof. Dr. Manuel Luis Martí ha sido designado como nuevo Presidente de la Academia Nacional de Medicina y es la primera vez que ese honor es concedido a un profesional de nuestra área.

Por último, deseamos transmitir un hondo pesar por la prematura desaparición de la Prof. Dra. Mercedes Traversa, quien tuvo una trayectoria destacada en nuestra sociedad y pudo reunir como pocos los atributos de excelencia académica y ejemplo de vida. Compartimos el privilegio de haberla conocido y el dolor de su pérdida.

\section{Dr. Julio César Bragagnolo}

Médico Especialista en Medicina Interna y Nutrición Jefe de la Unidad de Nutrición y Diabetes del Hospital J.M. Ramos Mejía Docente Asociado de la Universidad de Buenos Aires (UBA) 